Braz J Med Biol Res, December 2011, Volume 44(12) 1256-1260

doi: 10.1590/S0100-879X2011007500131

\section{Expression of the chemokine receptor CXCR4 on lymphocytes of leprosy patients}

V.A. Mendonça, G.E.B. Alvim de Melo, M.G. Araújo, V.O. Borges, R.D. Costa, O.A. Martins-Filho, A. Teixeira-Carvalho, R. Sathler-Avelar, M.M. Teixeira and A.L. Teixeira

The Brazilian Journal of Medical and Biological Research is partially financed by

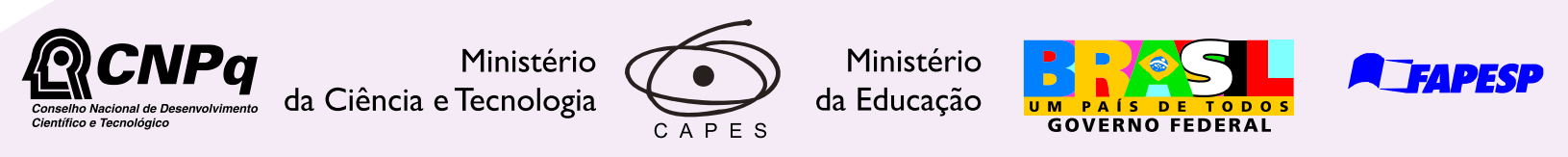

Institutional Sponsors
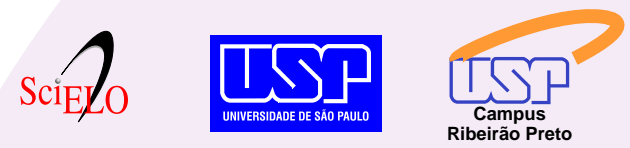

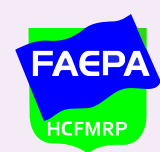

Ф SHIMADZU

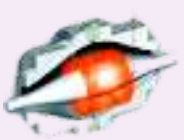

Explore High - Performance MS Orbitrap Technology In Proteomics \& Metabolomics

$\underset{\text { analitica }}{\text { analiticaweb.com.br }}$ SCIENTIFIC 


\title{
Expression of the chemokine receptor CXCR4 on lymphocytes of leprosy patients
}

\author{
V.A. Mendonça ${ }^{1,2 *}$, G.E.B. Alvim de Melo2*, M.G. Araújo ${ }^{3}$, V.O. Borges ${ }^{1}$, \\ R.D. Costa ${ }^{1}$, O.A. Martins-Filho ${ }^{4}$, A. Teixeira-Carvalho ${ }^{4}$, R. Sathler-Avelar ${ }^{4,5}$, \\ M.M. Teixeira ${ }^{1}$ and A.L. Teixeira ${ }^{1,3}$ \\ ${ }^{1}$ Laboratório de Imunofarmacologia, Instituto de Ciências Biológicas, \\ Universidade Federal de Minas Gerais, Belo Horizonte, MG, Brasil \\ ${ }^{2}$ Laboratório de Imunologia, Universidade Federal dos Vales do Jequitinhonha e Mucuri, \\ Diamantina, MG, Brasil \\ ${ }^{3}$ Departamento de Clínica Médica, Faculdade de Medicina, \\ Universidade Federal de Minas Gerais, Belo Horizonte, MG, Brasil \\ ${ }^{4}$ Centro de Pesquisas René Rachou, Fundação Oswaldo Cruz, Belo Horizonte, MG, Brasil \\ ${ }^{5}$ Centro Universitário Newton Paiva e Universidade Vale do Rio Verde/UninCor, Belo Horizonte, MG, Brasil
}

\begin{abstract}
Leprosy is caused by Mycobacterium leprae, which induces chronic granulomatous infection of the skin and peripheral nerves. The disease ranges from the tuberculoid to the lepromatous forms, depending on the cellular immune response of the host. Chemokines are thought to be involved in the immunopathogenesis of leprosy, but few studies have investigated the expression of chemokine receptors on leukocytes of leprosy patients. In the present study, we evaluated 21 leprosy patients (M/F: 16/5) with a new diagnosis from the Dermatology Outpatient Clinic of the University Hospital, Federal University of Minas Gerais. The control group was composed of 20 healthy members (M/F: 15/5) of the community recruited by means of announcements. The expression of CCR2, CCR3, CCR5, and CXCR4 was investigated by flow cytometry on the surface of peripheral blood lymphocytes. There was a decrease in percentage of CD3+CXCR4+ and CD4+CXCR4+ lymphocytes in the peripheral blood of leprosy patients (median [range], 17.6 [2.7-41.9] and 65.3 [3.9-91.9], respectively) compared to the control group (median [range], 43.0 [3.7-61.3] and 77.2 [43.6-93.5], respectively). The percentage of CD4+CXCR4+ was significantly lower in patients with the tuberculoid form (median [range], 45.7 [0.0-83.1]) of the disease, but not in lepromatous patients (median [range], 81.5 [44.9-91.9]). The CXCR4 chemokine receptor may play a role in leprosy immunopathogenesis, probably directing cell migration to tissue lesions in tuberculoid leprosy patients.
\end{abstract}

Key words: Leprosy; Chemokine receptors; CXCR4; Leukocytes

\section{Introduction}

Leprosy is a chronic granulomatous infection that mainly affects the skin and peripheral nerves. The damage to peripheral nerves results in sensory and motor impairment with characteristic deformities and disability $(1,2)$. Although leprosy is no longer a health problem in developed countries, it continues to affect millions of people in large parts of Asia, Africa, and Latin America. Brazil, Nepal and Timor-Leste account for about $17 \%$ of the new cases reported in 2007 and for $23 \%$ of the cases reported at the beginning of 2008 (3). The presentation of leprosy depends on the varying host immune response to Mycobacterium leprae. Individuals with tuberculoid leprosy display a strong cell-based immune response that controls bacterial proliferation and lesions, whereas patients with lepromatous leprosy lack specific cellular immunity, ending up with high mycobacterial loads and severe clinical manifestations (4).

Chemokines are potent leukocyte chemoattractants and are thought to be involved in mycobacterial diseases, including leprosy $(5,6)$. Chemokines and their receptors may be divided into four families (CXC, CC, C, and CX3C) on the basis of the pattern of cysteine residues in the ligands (here $\mathrm{C}$ represents cysteine and $\mathrm{X} / \mathrm{X} 3$ represents one or

Correspondence: V.A. Mendonça, Universidade Federal dos Vales do Jequitinhonha e Mucuri, Rodovia MGT 367, km 583, No. 5000, 39100-000 Diamantina, MG, Brasil. E-mail: vaafisio@hotmail.com

*These authors contributed equally to this study.

Received April 2, 2011. Accepted September 30, 2011. Available online October 14, 2011. Published November $28,2011$. 
three noncysteine amino acids). Cells respond to chemokines through a family of seven transmembrane $\mathrm{G}$ proteincoupled chemokine receptors (7). Recently, we investigated plasma levels of CCL2, CCL3, CCL11, CCL24, CXCL9, and CXCL10 in leprosy patients $(5,6)$ and found increased CCL11 levels in plasma of leprosy patients compared to non-infected individuals, suggesting that measurement of this chemokine could be useful to support the diagnosis of leprosy $(5,6)$. There was no significant difference in the expression of CC (CCR2 and CCR5) or CXC (CXCR2) chemokine receptors across the leprosy spectrum in one study (8), but little is known about the role of chemokine receptors in the pathogenesis of leprosy. In the present study, we performed an ex vivo analysis of the chemokine receptors associated with elevated chemokines in leprosy patients (CCL11 binds to CCR3) and those involved in migration of macrophages and lymphocytes, cells thought to be involved in the pathogenesis of leprosy. Therefore, levels of CCR2, CCR3, CCR5, and CXCR4 were evaluated on the surface of peripheral blood lymphocytes from leprosy patients and healthy individuals by flow cytometry.

\section{Subjects and Methods}

\section{Subjects}

Subjects included in this study were from the Dermatology Outpatient Clinic of the University Hospital, Federal University of Minas Gerais, Belo Horizonte, Brazil. Twentyone patients with a recent diagnosis of leprosy entered the study before treatment (mean age, years [range]: 44.3 [22.0-69.0]; M/F: 16/5). At baseline, patients underwent complete dermatological and neurological evaluations by a physician with expertise in leprosy management. Slit skin smears and skin lesion biopsies were collected at baseline and patients were classified according to Ridley-Jopling criteria (9). There were 14 tuberculoid leprosy (TT) patients and 7 lepromatous leprosy (LL) patients.

Twenty age- and gender-matched healthy individuals from the community were used as controls (mean age, years [range]: 39.5 [23.0-53.0]; M/F: 15/5). All subjects were seronegative for human immunodeficiency virus (HIV) and none presented other immunosuppressive conditions. The Ethics Committee (COEP) of the Universidade Federal de Minas Gerais approved the study and all patients gave written informed consent to participate.

\footnotetext{
Flow cytometry analysis of peripheral blood leukocytes

Biological samples consisting of $5 \mathrm{~mL}$ venous peripheral blood were collected using EDTA. The samples were collected by a trained professional at the outpatient clinic. After collection, the blood was used within $24 \mathrm{~h}$ for immunophenotypic analysis by flow cytometry.

White blood cell phenotypes were analyzed according to the procedure recommended by Becton Dickinson (USA), modified as follows: $100-\mu \mathrm{L}$ EDTA blood samples in $12 \mathrm{x}$
}

75-mm tubes were incubated in the dark with $1 \mu \mathrm{L}$ undiluted monoclonal antibodies (MAbs) specific for several cell surface markers (CD3 clone UCHT1, CD4 clone 11830, CD8 clone 37006, CCR3 clone 61828, labeled with FITC, CCR2 clone 48607, CCR5 clone CTC5, CXCR4 clone 12G5 labeled with PE, CD4 clone 920, CD8 clone 925 labeled with TC; R\&D Systems, USA) for 20-30 min at room temperature. After incubation, erythrocytes were lysed with $100 \mu \mathrm{L}$ lysing solution (Optlyse-B, Immunotech, France) for $5 \mathrm{~min}$, followed by the addition of $900 \mu \mathrm{L}$ distilled water and reincubation for 10 min. White blood cells were then washed twice with $1 \mathrm{~mL}$ phosphate-buffered saline (PBS) containing $0.01 \%$ sodium azide. Cell preparations were fixed in $500 \mu \mathrm{LFACS}$ fix solution (10 g/L paraformaldehyde, $10.2 \mathrm{~g} / \mathrm{L}$ sodium-cacodylate, 6.65 $\mathrm{g} / \mathrm{L}$ sodium-chloride). Cytofluorimetric data were acquired with a Becton Dickinson FACScan-instrument (Becton Dickinson, USA). The lymphocytes were assorted and gated based on their forward (FSC) and side (SSC) laser scatter properties. After lymphocyte selection, cells were analyzed for fluorescence properties and T lymphocytes were analyzed using single staining with an anti-CD3 monoclonal antibody. $\mathrm{T}$ lymphocyte subsets were identified using anti-CD4 and anti-CD3 double-staining for helper T cells while anti-CD8 and anti-CD3 double-staining was used for the identification of T cytotoxic lymphocytes. The expression of the chemokine receptors CCR2, CCR3, CCR5, and CXCR4 on helper T cells was found on CD4+ lymphocytes. For analysis of cytotoxic $T$ lymphocytes the chemokine receptor profile was evaluated on gated high CD8 lymphocytes.

\section{Statistical analysis}

Differences between two groups were determined by the Student $t$-test or the Mann-Whitney U-test for normally or non-normally distributed data, respectively. Differences among three groups were determined by ANOVAor the Kruskal-Wallis test in normally or non-normally distributed data, respectively. Statistical significance was set at $P<0.05$.

\section{Results}

\section{Analysis of total leukocyte cells and lymphocytes in peripheral blood}

There was no significant difference in total number of peripheral blood leukocytes between leprosy patients and healthy subjects. There was no difference in number or percentage of total lymphocytes, but there was a decrease in percentage of CD3+ T cells in the leprosy group (median [range], control $=67.2$ [39.5-78.9] and leprosy patients $=$ 58.2 [12.1-74.3], $\mathrm{P}=0.02$ ).

However, there was no significant difference in percentage of CD3+CD4+ (median [range], control = 42.1 [33.760.5] and leprosy patients $=39.0$ [24.3-55.0], $\mathrm{P}=0.19$ ), or in percentage of CD3+CD8+ T cells (median [range], control $=22.8$ [7.6-41.3] and leprosy patients $=19.1$ [10.138.0], $P=0.31$ ). 


\section{Analysis of expression of CCR2, CCR3, CCR5, and CXCR4 chemokine receptors}

There were no significant differences in the expression of CCR2, CCR3 or CCR5 chemokine receptors on peripheral blood lymphocytes from leprosy patients and healthy subjects. However, we observed that the percentage of CD3+CXCR4+ and CD4+CXCR4+T cells was significantly lower in the peripheral blood of leprosy patients compared to healthy subjects (Table 1).
Analysis of CXCR4 chemokine receptor on the lymphocyte surface of peripheral blood mononuclear cells of patients with the polar forms of leprosy

Next, we analyzed the expression of CXCR4 in patients with the polar forms of the disease, i.e., TT and LL patients. The percentage of CD3+CXCR4+ lymphocytes was significantly lower in TT and LL patients than in the control group (Figure 1A). The percentage of CD4+CXCR4+ was also significantly lower in TT patients compared to control

Table 1. Analysis of lymphocyte (T CD3, CD4, and CD8) expression of CCR2, CCR3, CCR5, and CXCR4 chemokine receptors in healthy controls $(N=20)$ and leprosy patients (LE; $N=21)$.

\begin{tabular}{|c|c|c|c|c|c|c|c|c|}
\hline & \multicolumn{2}{|c|}{ CCR2 } & \multicolumn{2}{|c|}{ CCR3 } & \multicolumn{2}{|c|}{ CCR5 } & \multicolumn{2}{|c|}{ CXCR4 } \\
\hline & Control & LE & Control & LE & Control & LE & Control & LE \\
\hline T CD3 & $9.2(1.2-25.4)$ & $6.4(1.7-30.0)$ & $9.8(0.5-29.5)$ & $10.4(2.4-30.2)$ & $9.5(1.9-18.2)$ & $9.9(3.0-25.5)$ & $43.0(3.7-61.3)$ & $17.6(2.7-41.9)^{*}$ \\
\hline $\mathrm{T}$ CD4 & $9.6(2.2-35.0)$ & $5.2(0.0-27.1)$ & $4.8(0.1-30.5)$ & $8.5(1.3-45.0)$ & $7.7(3.0-15.9)$ & $11.1(1.4-47.5)$ & $77.2(43.6-93.5)$ & $65.3(3.9-91.9)^{+}$ \\
\hline T CD8 & $17.5(0.6-62.9)$ & $15.9(1.8-70.2)$ & $27.3(2.6-82.2)$ & $18.6(0.9-64.5)$ & $23.6(1.6-65.7)$ & $27.8(2.2-53.3)$ & $49.2(4.7-94.7)$ & $51.2(5.2-98.5)$ \\
\hline
\end{tabular}

Data are reported as median (range). ${ }^{*} \mathrm{P}<0.001,{ }^{+} \mathrm{P}=0.01$, LE compared to control (Mann-Whitney U-test).

A

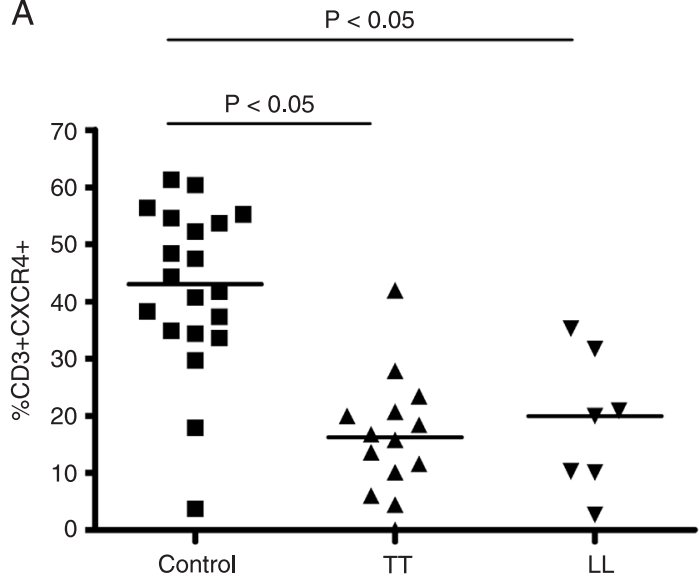

C

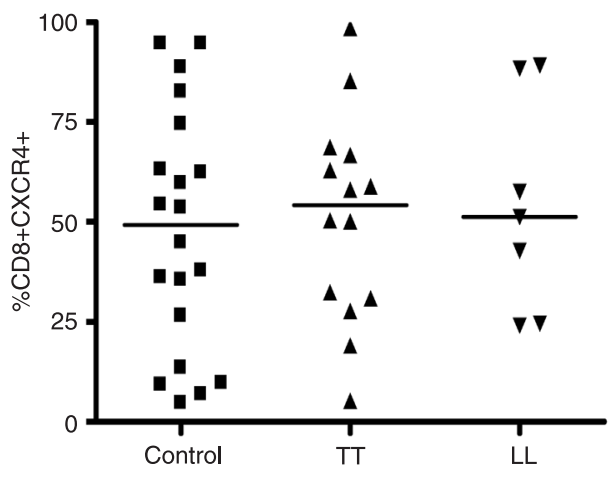

B

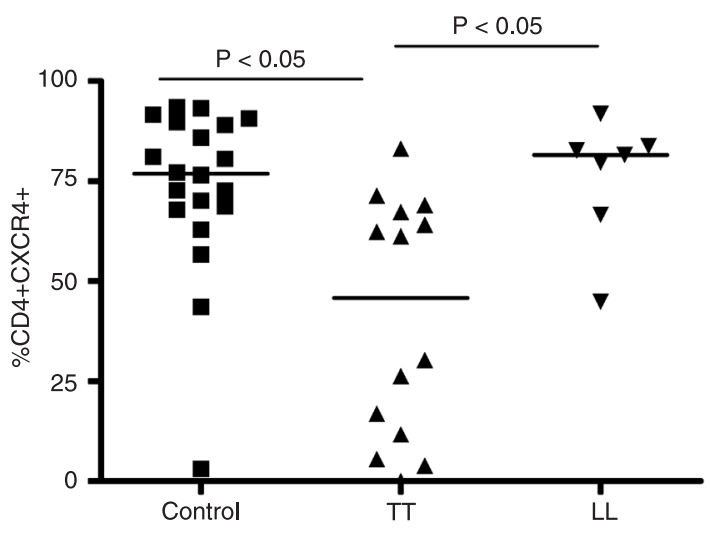

Figure 1. Percent analysis of CXCR4 chemokine receptor on the surface of peripheral blood lymphocytes of controls $(\mathrm{N}=20)$ and of patients with the polar form of leprosy. Tuberculoid leprosy (TT =14), lepromatous leprosy $(L L=7)$. Lymphocyte percentage of T $(A)$, TCD4 $(B)$, and TCD8 $(C)$. Horizontal lines indicate median values. $\mathrm{P}<0.05$ (KruskalWallis test). 
(Figure 1B). However, the percentage of CD4+CXCR4+ in LL patients was similar to that of the control group and greater than the percentages found in the TT group (Figure $1 \mathrm{~B})$. There was no difference in the expression of CCR2, CCR3 or CCR5 chemokine receptors on the surface of lymphocytes from control subjects and from patients with the polar forms of leprosy (data not shown).

\section{Discussion}

In the present study, we assessed by flow cytometry the expression of chemokine receptors on the surface of lymphocytes from leprosy patients. We found that the percentage of CD3+ cells, CD3+CXCR4+, and CD4+CXCR4+ lymphocytes were significantly lower in leprosy patients compared to healthy subjects. We also found that the percentage of CD4+CXCR4+ in TT patients was significantly lower than controls and LL patients.

There was no difference in percentage or number of CD4+ and CD8+ $T$ cells between leprosy patients and healthy subjects, but the percentage of $\mathrm{T}$ cells (CD3+ lymphocytes) was lower in leprosy patients. Similarly, a recent flow cytometry study of circulating leukocytes in pulmonary tuberculosis patients, an infectious disease also caused by Mycobacteria, showed that the percentage of CD3+ cells was lower than in control subjects (10). A previous study has demonstrated that activation of $T$ cells promotes down-regulation of the CD3 receptor (11). Since the leprosy patients included in this study were not treated at the time blood was collected, it is possible that T cells were activated in a chronic manner, as has been reported for tuberculosis. Such chronic activation may have accounted for the drop in CD3 expression but further studies with markers of $\mathrm{T}$ cell activation need to be performed to explain these differences.

Recently, we investigated the expression of CXCR4 on the surface of circulating leukocytes of leprosy patients and found no significant difference in the analysis of CXCR4 on the surface of TCD4 and TCD8 lymphocytes (12). In the present study, the percentage of CD3+CXCR4+ and CD4+CXCR4+ cells was decreased in leprosy patients compared to controls. Moreover, the percentage of CD4+CXCR4+ was lower in TT patients compared to LL patients. In the first study, all leprosy patients had the LL form of the disease, which may have masked the observations made in the present study. Indeed, in agreement with our previous results (12), levels of CXCR4 on CD4+ and CD3+ $T$ cells were similar for $L L$ and healthy subjects. Therefore, it seems that the lower CXCR4 levels observed in leprosy patients is accounted for mainly by the reduction in levels of these receptors on lymphocytes from patients with the TT polar form of the disease.

CXCR4, the receptor for CXCL12/stromal cell-derived factor 1a, is expressed on airway epithelial cells (13), naive T cells (14), and CD4+ Th2 cells (15). CXCL12 is often expressed constitutively in the absence of any stimuli, suggesting that CXCR4-CXCL12 interactions are involved in maintaining homeostasis. Studies have shown that, in addition to homeostasis, this pair: chemokine-chemokine receptor has been implicated in various diseases, including asthma (16), cancer (17) and arthritis (18). In 1999, Patterson et al. (19) demonstrated that, during HIV infection, IL-10 up-regulates CXCR4 expression by T cells. Shalekoff et al. (20) found an altered CXCR4 and CCR5 repertoire on peripheral leukocytes of patients with pulmonary tuberculosis and HIV-1 infection and suggested that an altered ability of X4 and R5 viruses to enter CD4-bearing cells and interfere with cellular trafficking may be an important feature of the pathogenesis of HIV-1 and tuberculosis. A strong Th1 response against $M$. leprae results in tuberculoid leprosy, with few lesions. Lepromatous patients are characterized by multibacillary disseminated disease and a lack of specific $T$ cell responses to $M$. leprae, and their lesions express a type 2 cytokine profile that promotes a humoral immune response and a progressive disease (1). CXCR4 is expressed in CD4+ Th2 cells (15) and in the present study TT patients showed a decreased CD4+CXCR4+ percentage in peripheral blood leukocytes compared to LL patients. This finding suggests that these cell types are present in lower percentages in the circulation of TT patients because they may have migrated to the leprosy tissue lesion. Such a possibility needs to be investigated by evaluating the expression of CXCR4 on T cells that migrate to the site of disease.

The first limitation of our study regards the sample size. Conversely, the stringent inclusion criteria may be regarded as a strength of the study. Second, we did not investigate the expression of chemokine receptors in leprosy skin lesions. This will be an important future task to be considered for correlation with the present findings.

This study has demonstrated the lower expression of CXCR4 on CD4+ T cells from patients with leprosy. The observation that reduced expression of CXCR4 occurs in TT patients, but not in those with the LL polar form of the disease, suggests that CXCR4 may play a role in driving the recruitment of lymphocytes to the skin. As TT patients have fewer microorganisms than those with the LL form, it is suggested that CXCR4 may have a role in the control of infection.

\section{Acknowledgments}

We thank the staff of the Dermatology Unit, University Hospital, UFMG, for recruiting leprosy patients and for their assistance. Research supported by CNPq and FAPEMIG, Brazil. 


\section{References}

1. Scollard DM, Adams LB, Gillis TP, Krahenbuhl JL, Truman RW, Williams DL. The continuing challenges of leprosy. Clin Microbiol Rev 2006; 19: 338-381.

2. Mendonça VA, Costa RD, Brito-Melo GEA, Antunes CM, Teixeira AL. Imunologia da hanseníase. An Bras Dermatol 2008; 83: 343-350.

3. WHO. Global leprosy situation 2008. Weekly Epidemiol Rec 2008; 83: 293-300.

4. Rinald A. The global campaign to eliminate leprosy. PLoS Med 2005; 2: 1222-1225.

5. Mendonca VA, Malaquias LC, Brito-Melo GE, CasteloBranco A, Antunes CM, Ribeiro AL, et al. Differentiation of patients with leprosy from non-infected individuals by the chemokine eotaxin/CCL11. Am J Trop Med Hyg 2007; 77: 547-550.

6. Mendonca VA, Costa RD, Lyon S, Penido RA, Borges VO, Bretas TL, et al. Plasma levels of chemokines during leprosy specific treatment. Acta Trop 2010; 113: 151-154.

7. Allen SJ, Crown SE, Handel TM. Chemokine: receptor structure, interactions, and antagonism. Annu Rev Immunol 2007; 25: 787-820.

8. Kirkaldy AA, Musonda AC, Khanolkhar-Young S, Suneetha $\mathrm{S}$, Lockwood DN. Expression of CC and CXC chemokines and chemokine receptors in human leprosy skin lesions. Clin Exp Immunol 2003; 134: 447-453.

9. Ridley DS, Jopling WH. Classification of leprosy according to immunity. A five-group system. Int J Lepr Other Mycobact Dis 1966; 34: 255-273.

10. Pokkali S, Das SD. Augmented chemokine levels and chemokine receptor expression on immune cells during pulmonary tuberculosis. Hum Immunol 2009; 70: 110-115.

11. Dutra WO, Martins-Filho OA, Cancado JR, Pinto-Dias JC, Brener Z, Freeman Junior GL, et al. Activated $T$ and $B$ lymphocytes in peripheral blood of patients with Chagas disease. Int Immunol 1994; 6: 499-506.

12. Mendonca VA, Melo GE, Teixeira MM, Martins-Filho OA, Antunes CM, Teixeira AL. Analysis of chemokine receptors on the surface of circulating leukocytes of individuals infected with Mycobacterium leprae: preliminary results. Rev Soc Bras Med Trop 2008; 41 (Suppl 2): 95-98.

13. Murdoch C, Monk PN, Finn A. Functional expression of chemokine receptor CXCR4 on human epithelial cells. Immunology 1999; 98: 36-41.

14. Sallusto F, Lenig D, Mackay CR, Lanzavecchia A. Flexible programs of chemokine receptor expression on human polarized T helper 1 and 2 lymphocytes. J Exp Med 1998; 187 : 875-883.

15. Jourdan $\mathrm{P}$, Abbal $\mathrm{C}$, Noraz N, Hori T, Uchiyama T, Vendrell $\mathrm{JP}$, et al. IL-4 induces functional cell-surface expression of CXCR4 on human T cells. J Immunol 1998; 160: 41534157.

16. Lukacs NW, Berlin A, Schols D, Skerlj RT, Bridger GJ. AMD3100, a CxCR4 antagonist, attenuates allergic lung inflammation and airway hyperreactivity. Am J Pathol 2002; 160: 1353-1360.

17. Payne AS, Cornelius LA. The role of chemokines in melanoma tumor growth and metastasis. J Invest Dermatol 2002; 118: 915-922.

18. Buckley CD, Amft N, Bradfield PF, Pilling D, Ross E, Arenzana-Seisdedos $\mathrm{F}$, et al. Persistent induction of the chemokine receptor CXCR4 by TGF-beta 1 on synovial T cells contributes to their accumulation within the rheumatoid synovium. J Immunol 2000; 165: 3423-3429.

19. Patterson BK, Czerniewski M, Andersson J, Sullivan Y, Su F, Jiyamapa D, et al. Regulation of CCR5 and CXCR4 expression by type 1 and type 2 cytokines: CCR5 expression is downregulated by IL-10 in CD4-positive lymphocytes. Clin Immunol 1999; 91: 254-262.

20. Shalekoff S, Pendle S, Johnson D, Martin DJ, Tiemessen CT. Distribution of the human immunodeficiency virus coreceptors CXCR4 and CCR5 on leukocytes of persons with human immunodeficiency virus type 1 infection and pulmonary tuberculosis: implications for pathogenesis. J Clin Immunol 2001; 21: 390-401. 